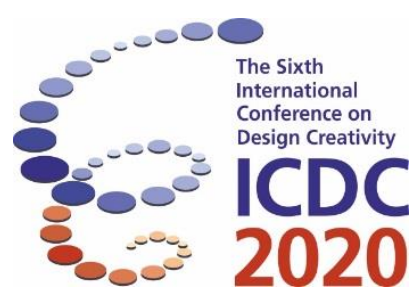

\title{
Brain Activity in Constrained and Open Design Spaces: An EEG study
}

\author{
Sonia Vieira ${ }^{1}$, John S. Gero ${ }^{2}$, Jessica Delmoral ${ }^{3}$, Shumin Li $^{1}$, Gaetano \\ Cascini $^{1}$, António Fernandes ${ }^{4}$ \\ ${ }^{1}$ Department of Mechanical Engineering, Politecnico di Milano, Italy \\ ${ }^{2}$ Department of Computer Science and School of Architecture, UNCC, Charlotte, NC, USA \\ ${ }^{3}$ INEGI-FEUP, Porto \\ ${ }^{4}$ Faculty of Engineering, University of Porto, Portugal
}

\begin{abstract}
Creativity is recognized as essential for changing the design space from constrained to open spaces. This study compares the neurophysiological activations of 18 professional industrial designers in two prototypical tasks, a problem-solving constrained layout task and an open design task. The analysis focused on measuring the cognitive demand in three stages of designing in constrained and open design spaces, namely: reading, problem-solving/reflection and layout/sketching. Results indicate significant differences in activations between the constrained task and the design task. Significant differences in activations involved in design reading, reflecting and sketching in open design tasks can be found in the left prefrontal cortex, temporal and occipital cortices. In particular, reading open or constrained requests evoked different levels of conceptual expansion prompting designers to change their design space, while reflecting evoked visual imagination and associative reasoning modes and hemispheric differences from problem-solving leading to expanded activation in sketching, which translates in higher activation in the open design task. These results show significantly different brain activations when designing in constrained and open design spaces.
\end{abstract}

Keywords: designing, open space, constrained space, electroencephalography, industrial designers

\section{Introduction}

Neural processes associated with general creativity have been widely investigated (Abrahams, 2019; Benedek et al., 2014; Dietrich \& Haider, 2017; Pidgeon et al., 2016). Higher neurophysiological activation is traditionally associated with conceptual expansion and implies a creative change in the approach to the request (Abrahams, 2019). However, investigations into design creativity related neural processes are still in their infancy. Designing, as an activity that is carried out over time entails a range of cognitive activities that can take minutes to unfold and generate solutions. A useful abstraction in understanding designing is the notion of design space, where designers explore an abstract space of possibilities (Amstel et al. 2016; MacLean et al. 2011) of which the problem-solving view of design claims that the designing process commences with an exploration within the problem space (Goel \& Pirolli, 1992; Goldschmidt, 1997) while others claim that designing commences by generating the solution space (Dorst, 2019; Dorst \& Cross 2001; Gero, 1990; Gero \& Kumar, 1993; Kruger \& Cross 2006; Visser 2009; Yoshikawa, 1981). Both views have been used in design cognition studies based on methods such as protocol analysis (Goldschmidt, 2014; Kan \& Gero, 2017). Another view is the notion 
that the design space can be constrained or open, depending on the design request's level of constraint and openness to creative exploration and that is the focus of the research reported in this paper. While a constrained design space is usually confined by specific requirements, an open design space expands by the introduction of new design variables leading to solutions which may not have been possible earlier. This can occur where constraints are in conflict and hence there are no feasible solutions and a better design is desired or when the designer introduces new variables as part of the design process. In both cases a new solution space emerges (Gero \& Kumar, 1993; Mose Biskjaer \& Halskov, 2013). Understanding how the brain produces higher order cognitive functions such as those involved in designing and disentangling its cognitive processes through neurophysiological measurements requires a macro perspective, by distinguishing phases or stages of designing.

Design studies based on functional magnetic resonance imaging (fMRI) started over a decade ago (Alexiou et al., 2009) with a controlled experiment reporting preliminary results on the distinction between designing and problem-solving. As designing is a temporal activity, measurements using electroencephalography (EEG) have started to play a role in design research due to its high temporal resolution, readily available software, reduction in the cost of equipment and lower need for specialized training.

Neurocognitive studies using EEG commenced more than 40 years ago (Martindale \& Hines, 1975) investigating cortical activation during multiple tasks. Some 20 years later a study on categorization tasks of experts and novices (Göker, 1997) made use of EEG in design research. In the last 10 years, single domain-related EEG design studies have been carried out (Liang et al., 2017, Liu et al., 2018, Liu et al., 2016, Nguyen \& Zeng, 2010).

EEG has a limited spatial resolution, however, it offers a high temporal resolution in the order of milliseconds in a portable device which makes it a more suitable tool than fMRI to investigate designing as a temporal activity. The present study uses a low-cost EEG device for measuring the totality of the unfolding temporal neurophysiological activations during constrained and open design spaces, of the experiment session of each participant. When compared to medical grade systems, the limitations of low-cost systems, namely physical stability, multiplexed acquisition of electrodes, lower space resolution and more data loss do not have significant effects on what we are measuring, where we are interested not in specific episodes but average behavior over time while performing certain activities. This device requires proper setup, reliable and good enough to report preliminary results. Although the low-cost EEG devices have lower signal to noise ratio potentially resulting in lower quality of the signals, the signal processing method and statistical approaches used in post-processing, compensate for these potential effects.

This paper describes a study from a larger research project whose goal is to investigate neurophysiological activation of designers across multiple design domains (Vieira et al. 2019). The study reported in this paper is based on the analysis of industrial designers' neurophysiological activations using an EEG headset in the context of performing a constrained layout task and an open design task in a laboratory setting. The aim of this study is:

- investigate the neurophysiological activation differences of industrial designers when designing for constrained layout task and designing for an open task.

We explore the observable differences of the neurophysiological activations between the constrained space of a problem-solving request and an open design task based on free-hand sketching request. The analysis focuses on the neurophysiological activation differences observed along the different stages of the execution of the tasks. By temporally segmenting these activations for each participant, it is possible to distinguish brain activation across design sessions. We investigate the following research question:

- What are the differences in the neurophysiological activations of the cognitive demand of industrial designers when reading, problem-solving and laying out in a constrained design task, and reading, reflecting and sketching in an open design task?

\section{Methods}

The research question is investigated by using the constrained design task as the control/reference and statistically comparing the open design task with the reference task. In this study we compare absolute values known as transformed power (Pow), where the constrained problem-solving task's 
neurophysiological activation is the base for comparison. We report on the analysis of 29 experiment sessions. The tasks and experimental procedure (Vieira et al., 2019) were piloted prior to the full study, through five different sessions which produced changes resulting in the final experiment design.

\subsection{Participants}

The experimental sessions comprised 29 industrial designers. After eliminating 5 sessions where there were problems in the data collection and 6 whose data were outliers (as described in Section 2.5), we were left with 18 data sets. Results are based on 18 right-handed participants, all professional, aged 25$43(\mathrm{M}=31.7, \mathrm{SD}=7.3), 10$ men (age $\mathrm{M}=35.1, \mathrm{SD}=7.2)$ and 8 women (age $\mathrm{M}=27.5, \mathrm{SD}=5.1)$. The participants are all professionals (experience $\mathrm{M}=7.8, \mathrm{SD}=5.6$ ). This study was approved by the local ethics committee of the University of Porto.

\subsection{Experiment Tasks Design}

We adopted and replicated the constrained task based on problem-solving layout design described in the Alexiou et al. (2009) fMRI-based study. We matched the constrained task in terms of requests, number of constraints, stimuli and number of instructions. This task is considered a problem-solving task as the problem itself is well-defined, and the set of solutions is unique (Alexiou et al., 2009). We designed a block experiment which consisted of a sequence of tasks previously reported (Vieira et al., 2019). We added an open design task that included free hand sketching. This task is an ill-defined and fully unconstrained task unrelated to formal problem-solving. For this paper the focus is on the neurophysiological activations of both the constrained design task based on problem-solving and the open design task based on free hand sketching. We looked into total task activations and three stages corresponding to reading, problem-solving and layout stages of the first task, and reading, reflecting and sketching stages of the second task, Table 1.

Table 1 Description of constrained and open design spaces tasks.

\begin{tabular}{c|c}
$\begin{array}{c}\text { Constrained design Task } \\
\text { based on Problem-solving }\end{array}$ & $\begin{array}{c}\text { Open design Task } \\
\text { based on design sketching }\end{array}$ \\
\hline $\begin{array}{c}\text { In the first task the design of a set of furniture is available } \\
\text { and three conditions are given as requirements. The task } \\
\text { consists of placing the magnetic pieces inside a given area } \\
\text { of a room with a door, a window and a balcony. }\end{array}$ & $\begin{array}{c}\text { In the free-hand sketching task, the participants are asked } \\
\text { to: propose and represent an outline design for a future } \\
\text { personal entertainment system }\end{array}$ \\
\hline
\end{tabular}

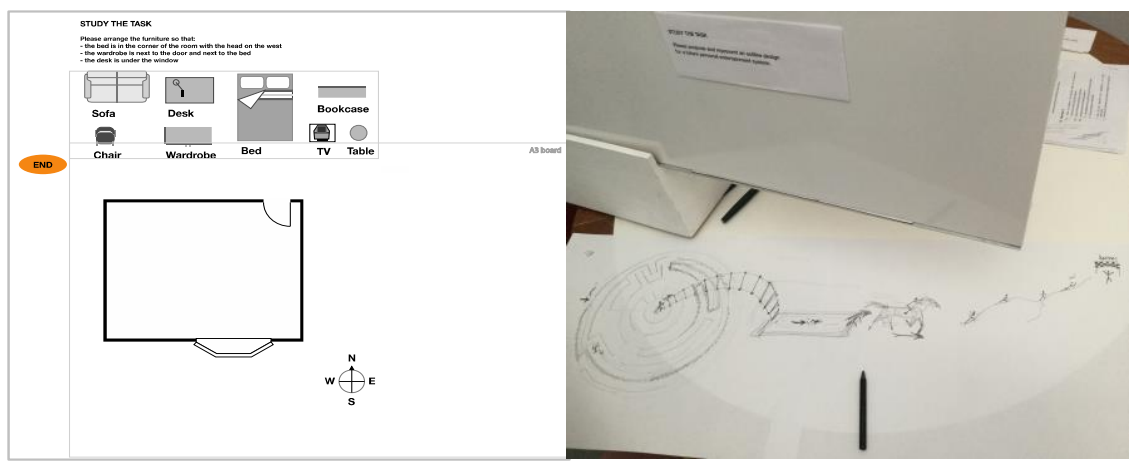

Figure. 1 Depiction of the problem-solving task and open free hand sketching design task.

Each participant was given two sheets of paper (A3 size) and three instruments, a pencil, graphite and a pen. The second task requires defining the problem and the solution spaces.

\subsection{Setup and Procedure}

A tangible interface for individual task performance was built based on magnetic material for easy handling. Differently from the original tasks (Alexiou et al., 2009), the magnetic pieces were placed at the top of the vertical magnetic board to prevent signal noise due to eye and head horizontal movements. 
The setup, full sequence of tasks and complete procedure is described elsewhere (Vieira et al. 2019). Electromagnetic interference of the room was checked for frequencies below $60 \mathrm{~Hz}$. One researcher was present in each experiment session to instruct the participant and to check for recording issues. A period of 10 minutes for setting up and a few minutes for a short introduction were necessary for informing each participant, reading and signing of the consent agreement and to set the room temperature. The researcher checked metallic accessories for electromagnetic interference and contact lenses. The researcher sat each participant at the desk and checked their posture. The researcher followed a script to conduct the experiment so that each participant was presented with the same information and stimuli. The participants were asked to start by reading the task request which took an average of 10s. The participants were asked to stay silent during the tasks and use the breaks for clarifying questions.

\subsection{Data Collection Methods}

EEG activity was recorded using a portable 14-channel system Emotiv Epoct. Electrodes are arranged according to the 10-10 I.S. Each of the Emotiv Epoc+ channels collects continuous signals of electrical activity at their location. The participants performed the tasks on the physical magnetic board, with two video cameras capturing the participant's face and activity. All the data captures were streamed using Panopto software (https://www.panopto.com/). Sessions took place at the University of Porto, between March and July of 2017 and June and September of 2018 in the Design Hub of Mouraria, Lisbon, during August 2018 between 9:00 and 15:00.

\subsection{Data Processing Methods}

The fourteen electrodes were disposed according to the 10-10 I.S, $256 \mathrm{~Hz}$ sampling rate, low cutoff 0.1 $\mathrm{Hz}$, high cutoff $50 \mathrm{~Hz}$. We adopted the blind source separation (BSS) technique based on canonical correlation analysis (CCA) for the removal of muscle artifacts from EEG recordings (De Clercq, 2006; Vos et al. 2010) adapted to remove the short EMG bursts, attenuating the muscle artifact contamination of the EEG recordings. A complete explanation of the BSS-CCA method is described elsewhere (Vieira et al. 2019). Data processing includes the removal of Emotiv specific DC offset with the Infinite Impulse Response (IIR) filter and BSS-CCA. Data analysis included total and band power values on individual and aggregate levels using MatLab and EEGLab open source software. All the EEG segments of the recorded data were used for averaging throughout the entire tasks and segments corresponding to each of the above-mentioned stages in analysis. The motor actions involved in the tasks using the tangible interface and the free hand sketching and their corresponding EEG signals are of the same source, thus we claim that the BSS-CCA procedure filters the signal of both from artifacts. We report on one measurement, the total signal power (Pow). The Pow is the transformed power, more specifically the mean of the squared values of microvolts per second $(\mu \mathrm{V} / \mathrm{s})$ for each electrode processed signal per task and per each stage. This measure tells us about the amplitude of the signal per channel and per participant magnified to absolute values. We present Pow values on aggregates of participants' individual results, per total task and for each stage.

We processed the Pow measurement for each participant. After a z-score was conducted to determine outliers, the criteria for excluding participants were based on the evidence of 6 or more threshold z-score values above 1.96 or below -1.96 and individual measurements above 2.81 or below -2.81 . Then, we calculated the mean and standard deviation of each measure at the aggregate level. Amplitudes greater than 2.5 standard deviations from the mean as threshold values were excluded per channel. In this process 11 further sessions were excluded leaving 18 data sets for the analysis.

We divided each task in three stages per participant data. The first stage is the reading period of both tasks. Then for the constrained problem-solving task, we divide the stage in which participants strictly answer to the request of locating three pieces of furniture, from the rest of the task in which participants continue the layout design task. For the open design task a division is made between the reflecting stage participants take before starting to sketch, and the sketching stage.

\subsubsection{Statistical approach}

We performed standard statistical analyses based on the design of the experiment: a repeated-measures design with pairwise comparisons to follow up on specific differences with task, hemisphere and electrode 
as within-subject factors and effect size through Cohen's $d$. These analyses were performed for the dependent variable of Pow and for all the within-subject variables. The threshold for significance in all the analyses is $p \leq .05$. To compare the Pow of the two tasks we performed an analysis by running a $2 \times 2 \times 7$ repeated-measurement ANOVA, with the within-subject factors of task, hemisphere and electrode. To compare the Pow of the three stages of each task we performed an analysis by running a $3 \times 2 \times 7$ repeatedmeasurement ANOVA, with the within-subject factors of task, hemisphere and electrode.

\section{Analysis of Results}

Results of total transformed power (Pow) across the 18 professional industrial designers indicate that the tasks can be distinguished from each other (Vieira et al., 2019).

\subsection{Transformed Power of the Constrained and the Open Tasks}

Total transformed power (Pow), for each task across the 14 channels are depicted in Figure 2. The plot shows the two hemispheres by distributing the electrodes (10-10 IS) symmetrically around a vertical axis. Pow scores per electrode (average of the entire task) can be considered by comparing with the vertical scale and across the two tasks. Results from running the ANOVA, revealed main effects for task $(p<.001)$, hemisphere $(p<.001)$ and electrode $(p<.001)$.

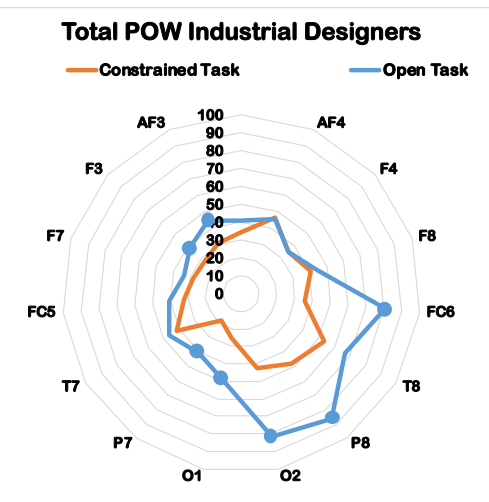

Figure 2 Transformed power (Pow) and channels of significant differences between the constrained design task and the open design task highlighted with a solid circle.

The open design task based on sketching shows higher neurophysiological activation than the constrained design task based on problem-solving. Below we report on significant $(p \leq .05)$ differences. The solid circles indicate significant differences from the pairwise comparisons that were conducted to follow up on the main effects, Figure 2. Results indicate significant differences in activations between the two tasks. The expanded activation in the open design task, which is traditionally associated with conceptual expansion (Abrahams, 2019) implies a creative change in the approach to the request. The results of total power across tasks encouraged further investigation to discriminate between main stages of activities in each task. We further report results on segmenting the data signal according to the stages explicit in each task. The analysis focused on stages of the constrained and open design, namely, reading and problem-solving or reflection and layout or sketching. We look at the cognitive demand in constrained and open design and how it translates into brain activation.

\subsection{Analysis of Transformed Power of the Stages of the Constrained Space Design Task}

The stages of the constrained design task considered were: reading the task request, solving the problemsolving specific request, and the remainder of the task, in case participants decide to place more pieces of furniture, i.e. layout. Total transformed power (Pow), for each stage across the 14 channels are depicted in Figure 3(a). The plot shows the two hemispheres by distributing the electrodes (10-10 IS) symmetrically around a vertical axis. Pow scores per electrode (average of the entire stage) can be considered by comparing with the vertical scale and across the three stages. The constrained design task shows small differences in the neurophysiological activation between the three stages. The last stage, in which participants have more freedom, unexpectedly shows lower activation across channels, except for 
channels FC5 and T7, denoting how participants' activations decreased during the layout stage of the constrained design request.

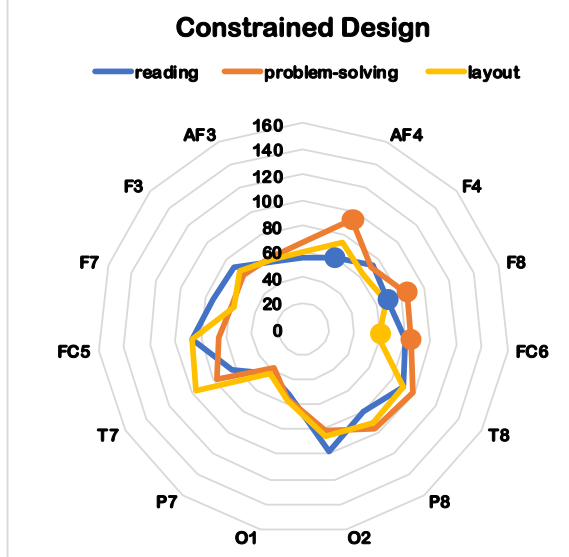

(a)

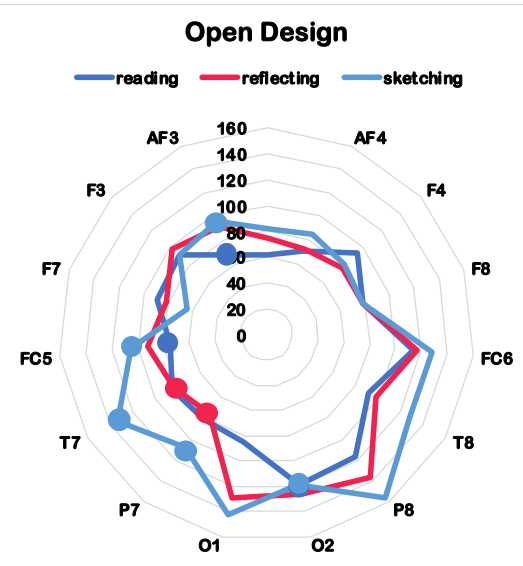

(b)

Figure 3 Transformed power (Pow) across channels between the stages of (a) reading, problemsolving and layout of constrained design task and (b) reading, reflecting and sketching of the open design task.

Results from running the ANOVA show no significant main effects between the stages of the constrained design task. The solid circles indicate significant differences from the pairwise comparisons $(p \leq .05)$ between channels across stages, Figure 3(a). The channels AF4 $(p<.01)$ and F8 ( $p=.02)$ show significant differences between reading and problem-solving, and the channel FC6 ( $\mathrm{p}=.03)$ shows significant differences between problem-solving and layout.

\subsection{Analysis of Transformed Power of the Stages of the Open Space Design Task}

The stages of the open design task considered were: reading the task request, reflecting about the request, and sketching. Four participants included writing activities, brain mapping, schematics and writing key words in the stage before sketching. The neurophysiological results from these activities were excluded from this comparison. Total transformed power (Pow), for each stage across the 14 channels are depicted in Figure 3(b). The open design task shows differences in the neurophysiological activation between the three stages. Results from running the ANOVA show no significant main effects between the stages. However, there is a three-way interaction effect between the factors task, hemisphere and electrode $(p=.01)$. The solid circles indicate significant differences from the pairwise comparisons $(p \leq .05)$, Figure 3(b). The channels O2 ( $p=.03)$, FC5 ( $p=.04)$ and AF3 ( $p<.05)$ show significant differences between reading and sketching, and the channels P7 $(p<.05)$ and T7 ( $p \leq .05)$ show significant differences between reflecting and sketching.

\subsection{Analysis of Transformed Power of Categorical Stages between the Design Tasks}

Three stages of categorical similarity between the tasks were considered for comparison namely, reading, problem-solving/reflecting and layout/sketching. Total transformed power (Pow), for each comparison across the 14 channels are depicted, Figure 4. Cohen's $d$ was calculated to measure the effect size for each electrode transformed power (Pow), between the stages, Table 2. The solid circles indicate channels of moderate (>.50) and large (>.80) effect size in Figure 4.

Table 2 Cohen's $d$ for the channels between categorical stages.

\begin{tabular}{c|c|c|c|c|c|c|c|c|c|c|c|c|c|c|c}
\hline & AF3 & F3 & F7 & FC5 & T7 & P7 & O1 & O2 & P8 & & T8 & FC6 & F8 & F4 & AF4 \\
\hline Reading & & .51 & & & & .91 & .74 & .65 & .69 & & & .72 & & & \\
\hline Problem-solving/Reflecting & .65 & .77 & .51 & .63 & & 1.24 & 1.89 & 1.25 & .81 & & & .58 & & & \\
\hline Layout/Sketching & .84 & .61 & & & .62 & 1.65 & 2.03 & .83 & 1.39 & & .89 & 2.02 & & & \\
\hline
\end{tabular}




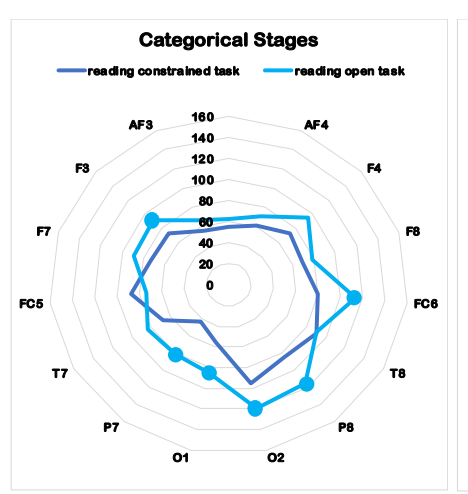

(a)

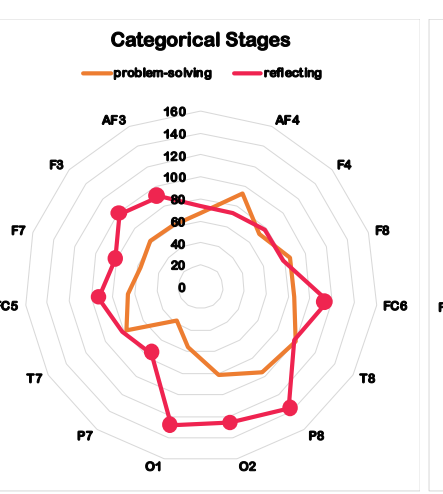

(b)

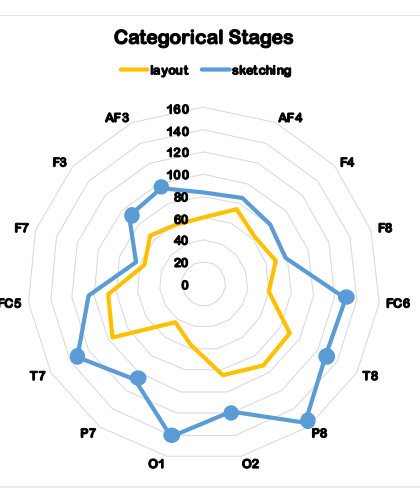

(c)

Figure 4 Transformed power (Pow) across channels between the stages of (a) reading, (b) problemsolving and reflecting and (c) layout and sketching between tasks.

\section{Discussion and Conclusion}

Results show significant differences between the tasks as reported previously (Vieira et al., 2019) and in the neurophysiological activations of some channels of the industrial designers across the different stages of the constrained and open design tasks. While the constrained design task shows significant differences between its three stages in channels of the right prefrontal cortex, the open design task revealed significant differences in the opposite left hemisphere and across the occipitotemporal cortex. The neurophysiological activations in the brains of these designers showed a higher level of activation in the open design task compared to the constrained design task. From the results of statistically comparing categorical stages between the tasks, we can infer the following:

- Reading constrained or open requests can evoke different levels of conceptual expansion, prompting designers to change their solution space, which translates in expanded activation in the open design task. This has important implications for the understanding of design as it supports the claim that it is the way designers formulate their understanding of the requirements given to them that plays a significant role in everything that follows.

- Problem-solving a constrained task and reflecting about an open task, revealed hemispheric differences with expanded activation in the open design task. Visual imagination and associative cognitive functions of the occipital and temporal cortices and inductive and deductive reasoning in left hemisphere seem to support designers expanding the design space.

- Sketching in the open design task shows higher amplitudes when compared to the layout stage, possibly affected by fixation in the constrained task. Cognitive functions of the temporal and occipital cortices channels with highest activations seem to play a role in sketching.

Significant differences in activations when reading the task, reflecting and sketching in open design tasks found in the left prefrontal cortex, temporal and occipital cortices are consistent with results from studies on new creative ideas (Benedek et al., 2014). Taking the approach that creativity is associated with opening the space of possible designs, amongst other changes, this experiment has shown that neurophysiological activations can be used as a measure of the change and expansion in design spaces. We asked participants to design for a highly constrained task which, we infer, results in a constrained design space and then to design for an open task which results in an open design space.

\section{Acknowledgments}

The research is funded by the Portuguese Foundation for Science and Technology, grant number SFRH/BPD/104281/2014. The second author has been supported in part by a grant from the US National Science Foundation, grant number EEC-1929896.

\section{References}

Abrahams, A. The Neuroscience of Creativity, Cambridge, UK, Cambridge University Press. 2019. 
Alexiou, K, Zamenopoulos, T, Johnson, J.H, Gilbert S.J. "Exploring the neurological basis of design cognition using brain imaging: some preliminary results.” Design Studies. Vol.30 No.6. 2009: 623-647

Amstel, F. van, Hartmann, T., Voort, M., Dewulf, G. "The social production of design space." Design Studies. Vol.46. 2016: 199-225.

Benedek, M; Jauk, E, Fink, A; Koschutnig, K·, Reishofer. G., Ebner. F', Neubauer, A. "To create or to recall? Neural mechanisms underlying the generation of creative new ideas." NeuroImage. Vol.88. 2014: 125-133.

De Clercq, W., Vergult, A., Vanrumste, B., Van Paesschen, W., Van Huffel, S. "Canonical correlation analysis applied to remove muscle artifacts from the electroencephalogram." IEEE Transactions on Biomedical Engineering. Vol.53. 2006: 2583-2587.

Dietrich, A., Haider, H. “A neurocognitive framework for human creative thought. Frontiers in Psychology, 2017. Vol.7:2078.

Dorst, K. "Co-evolution and emergence in design.” Design Studies. Vol.65. No. C, 2019: 65-77.

Dorst, K. \& Cross, N. "Creativity in the design process: Co-evolution of problem-solution." Design Studies. Vol.22. 2001: 425-437.

Gero, J. "Design Prototypes: a knowledge representation schema for design." Artificial Intelligence Magazine. Vol.11. No.4. 1998: 26-36.

Gero, J., Kumar, B. "Expanding design spaces through new design variables.” Design Studies. Vol.14. No.2. 1993: 210-221.

Goel, V., Pirolli, P. “The structure of design problem spaces.” Cognitive Science. Vol.16. 1992: 395-429.

Goldschmidt, G. "Capturing indeterminism: representation in the design problem space.” Design Studies Vol.22. 1997: 425-437.

Goldschmidt, G. Linkography: Unfolding the Design Process. MIT Press. 2014.

Visser, W. "Design: one, but in different forms." Design Studies. Vol.30 No.3. 2009: 187-223.

Göker, M. "The effects of experience during design problem solving." Design Studies. Vol.18. 1997: 405-426.

Nguyen. T. A., Zeng, Y. "Analysis of design activities using EEG signals." Volume 5: 22nd International Conference on Design Theory and Methodology. 2010: 277-286.

Kan, J.W.T., Gero, J.S. Quantitative Methods for Studying Design Protocols. Springer. 2017.

Kruger, C., Cross, N. Solution driven versus problem driven design: Strategies and outcomes. Design Studies. Vol.27 No.5. 2006: 527-548.

Liang, C., Lin, C., Yao, C., Chang, W., Liu, Y., Chen, S. "Visual attention and association: An electroencephalography study in expert designers.” Design Studies. Vol.48. 2017:76-95.

Liu, L., Nguyen, T., Zeng, Y., Ben Hamza, A. "Identification of relationships between electroencephalography (EEG) bands and design activities." Vol.7: 28th International Conference on Design Theory and Methodology. Charlotte, North Carolina, USA, August 21-24, 2016.

Liu, L., Li, Y., Xiong, Y., Cao, J., Yuan, P. 2018.“An EEG study of the relationship between design problem statements and cognitive behaviors during conceptual design." Artificial Intelligence for Engineering Design, Analysis and Manufacturing. Vol.32. 2018:351-362.

MacLean, A., Young, R., Bellotti, V., Moran, T. "Questions, options, and criteria: Elements of design space analysis". Human-Computer Interaction. Vol.6. No.3-4. 1991: 201-250.

Martindale, C., Hines, D. "Creativity and cortical activation during creative, intellectual and EEG feedback tasks." Biological Psychology. Vol.3. 1975: 91-100.

Mose Biskjaer; M. Halskov, K. "Decisive constraints as a creative resource in interaction design." Digital Creativity. Vol.25. N0.1. 2013: 27-61.

Pidgeon, L., Grealy, M., Duffy, A., Hay, L., McTeague, C., Vuletic, T., Coyle, D., Gilbert, S. "Functional neuroimaging of visual creativity: A systematic review and meta-analysis." Brain and Behavior. Vol.6 No.10. 2016:1-26.

Vieira, S., Gero, J., Delmoral, J., Gattol, V., Fernandes, C., Fernandes, A. “Comparing the design neurocognition of mechanical engineers and architects: A study of the effect of designer's domain", in Proceedings of the 22nd International Conference on Engineering Design, Delft, 5-8 August 2019. doi:10.1017/dsi.2019.191.

Vos, D., Riès, S., Vanderperren, K., Vanrumste, B., Alario, F., Huffel, V., Burle, B. "Removal of muscle artifacts from EEG recordings of spoken language production." Neuroinform. Vol.8. 2010:135-150.

Yoshikawa, H. (1981). "General design theory and a CAD system". In Sata, T. and Warman, E., editors, ManMachine Communication in CAD/CAM, Proceedings of The IFIP WG5.2-5.3 Working Conference 1980 (Tokyo), pages 35-57, North-Holland, Amsterdam. 